\title{
LETTERS \\ Describing Failure in a Clinical Clerkship
}

\author{
Flint Y. Wang, MD, Kathleen O. Degnan, MD, and Eric N. Goren, MD \\ University of Pennsylvania School of Medicine, Hospital of the University of Pennsylvania, Philadelphia, PA, USA.
}

J Gen Intern Med 32(4):378

DOI: $10.1007 / \mathrm{s} 11606-016-3979-5$

(C) Society of General Internal Medicine 2017

I "Describing Failure in a Clinical Clerkship," Nixon et al. ${ }^{1}$ examined competency deficiencies of 43 students who failed the internal medicine clerkship at the University of Minnesota School of Medicine. The authors categorized deficiencies using the domains of the Physician Competencies Reference $\mathrm{Set}^{2}$ designed by the American Association of Medical Colleges, which included medical knowledge, patient care, practice-based learning and improving, interpersonal and communication skills, professionalism, systems-based practice, inter-professional collaboration, and personalprofessional development. Surveys of residency program directors $^{3}$ frequently cite organization and efficiency as concerning deficiencies in residents, while this topic is largely overlooked in medical students. There is a perception that because medical students are just learning how to assess patients that organization and efficiency are skills that will just be obtained with more experience. The organization and efficiency categories rarely become an explicitly stated competency, and very few papers detail a focus on efforts to remediate within this domain. We propose that the missing links that tie together most of the attributes of learners failing internal medicine clerkships are actually organization and efficiency.

Coaching a learner through organization and efficiency allows the learner to apply knowledge and skills through a practical framework of what he/she should be doing minuteto-minute as they work up a new patient admission within the time constraints of patient care while applying aspects of other domains. Organization and efficiency deficiencies can significantly cloud the evaluator's assessment because when not addressed, they prevent showcasing of progress in other coached domains. We feel that organization and efficiency are at the root of nearly all deficiencies listed by Nixon et al. including: incomplete differential diagnosis, poor patient presentation skills, inadequate synthesis and application of knowledge, examination failure, inability to manage expected patient volume, poor communication with the team, delayed response to pages, and being late. If students do not approach patient care with a step-by-step organized framework and prioritization skills, they are less likely to deliver a poised and confident delivery of a clinical assessment leading to frustration from preceptors and perhaps inaccurate assessment of deficiencies in the other domains.

The lack of emphasis on organization and efficiency in the competency guideline creation process and in prior coaching literature plays a major role in the critical evaluation of remediation programs to help struggling medical students, especially for those who have not improved with coaching in other domains.

Corresponding Author: Flint Y. Wang, MD; University of Pennsylvania School of Medicine, Hospital of the University of Pennsylvania, Philadelphia, PA, USA (e-mail: flint.wang@uphs.upenn.edu).

Compliance with Ethical Standards:

Prior Presentations: None.

Conflict of Interest: The authors declare that they have no conflict of interest.

Funding Sources: None.

\section{REFERENCES}

1. Nixon LJ, Gladding SP, Duffy BL. Describing failure in a clinical clerkship: implications for identification, assessment and remediation for struggling learners. J Gen Intern Med. 2016;31:1172-1179.

2. Englander R, Cameron T, Ballard AJ, Dodge J, Bull J, Aschenbrener CA. Toward a common taxonomy of competency domains for the health professions and competencies for physicians. Acad Med. 2013;88:10881094.

3. Dupras DM, Edson RS, Halvorsen AJ, Hopkins RH Jr, McDonald FS. "Problem residents": prevalence, problems and remediation in the era of core competencies. Am J Med. 2012;125(4):421-425. 